\title{
Rotational and Translational Components of Motion Parallax: Observers' Sensitivity and Implications for Three-Dimensional Computer Graphics
}

\author{
Mary K. Kaiser and Michael J. Montegut \\ National Aeronautics and Space Administration
}

\author{
Dennis R. Proffitt \\ University of Virginia
}

\begin{abstract}
The motion of objects during motion parallax can be decomposed into 2 observer-relative components: translation and rotation. The depth ratio of objects in the visual field is specified by the inverse ratio of their angular displacement (from translation) or equivalently by the inverse ratio of their rotations. Despite the equal mathematical status of these 2 information sources, it was predicted that observers would be far more sensitive to the translational than rotational component. Such a differential sensitivity is implicitly assumed by the computer graphics technique billboarding, in which 3-dimensional (3-D) objects are drawn as planar forms (i.e., billboards) maintained normal to the line of sight. In 3 experiments, observers were found to be consistently less sensitive to rotational anomalies. The implications of these findings for kinetic depth effect displays and billboarding techniques are discussed.
\end{abstract}

Since von Helmholtz's discussions almost a century ago (von Helmholtz, 1910/1962), psychologists have been aware of the spatial layout information provided by motion parallax. Typically, however, descriptions of this information focus on the linear velocities (or displacements) of objects in the visual field. But as shown in Figure 1, the - relative depth of objects is specified equally well by the ratio of the angular velocities (or rotations) of the objects relative to the observer.

Recent psychophysical studies have demonstrated that observers are reasonably sensitive to angular velocities; discrimination thresholds for linear and angular velocities are of the same order of magnitude, although somewhat higher for angular motion (Kaiser, 1990; Kaiser \& Calderone,

Mary K. Kaiser and Michael J. Montegut, National Aeronautics and Space Administration (NASA) Ames Research Center; Dennis R. Proffitt, Department of Psychology, University of Virginia. Michael J. Montegut is a National Research Council Resident Associate.

Correspondence concerning this article should be addressed to Mary K. Kaiser, NASA Ames Research Center, Mail Stop 262-2, Moffett Field, California 940351000. Electronic mail may be sent via Internet to Mary K. Kaiser at moose@eos.arc.nasa.gov.
1991). Thus, in principle, observers could use either the translational or rotational component of motion parallax as a robust cue (or invariant) to relative depth.

That observers might be insensitive to the rotational component of motion parallax is implicitly assumed by a rendering technique used in computer simulations of visual environments (i.e., real-time, three-dimensional [3-D] graphics). This technique, termed billboarding, reduces a $3-\mathrm{D}$ object to a 2-D texture mapped image (i.e., a billboard) that is maintained normal to the observer's eye point. The computational savings of this technique are impressive; a complex object can be reduced to a single, texture-mapped polygon. However, the technique's efficacy is dependent on the observer's not noticing the object's failure to undergo canonical observer-relative rotations. Generally, database designers have assumed that billboarding is likely to be most successful for generally radially symmetric objects (e.g., trees). Such objects do not undergo boundary deformations during rotations; thus, it is assumed that the lack of rotation would be less noticeable (Foley, van Dam, Feiner, \& Hughes, 1990). However, this assumption has never been explicitly tested, and other factors that might affect the technique's effective- 


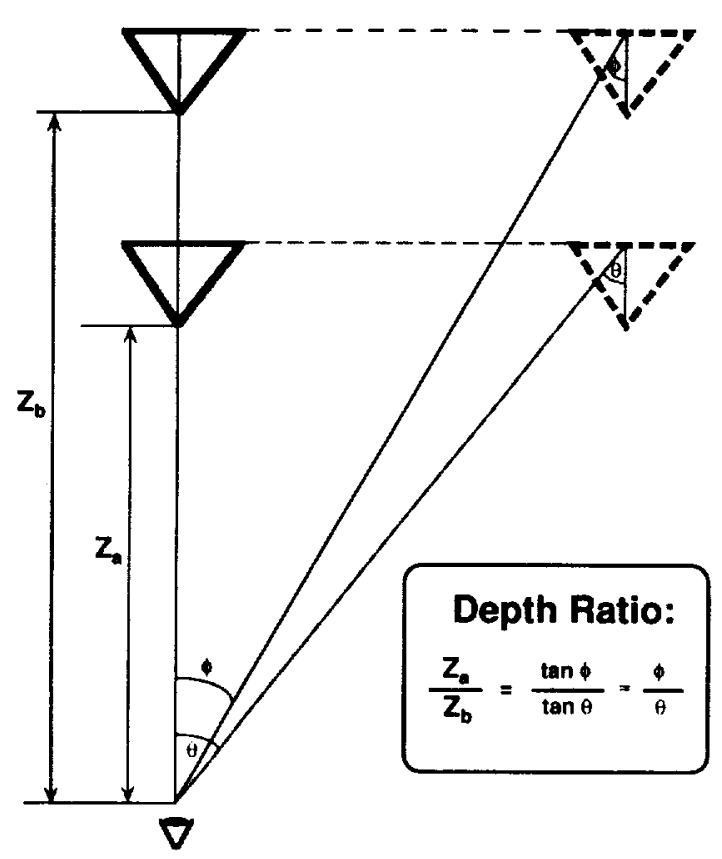

Figure 1. Rotational and translational components of motion parallax. When the two triangles move relative to the observer, the relative depth of the objects $\left(Z_{a} / Z_{b}\right)$ is specified both by the ratio of angular displacements of the objects in the observer's field of view $(\tan \phi / \tan \theta)$, and by the extent of observer-relative rotation (also [tan $\phi / \tan \theta])$.

ness (e.g., the distance of the object from the observer) have not been systematically explored. If it can be demonstrated that people are relatively insensitive to the observer-relative rotations that result from motion parallax, such findings validate the utility of graphical rendering techniques that omit (or simplify) rotational image transformations and the substantial computational savings these techniques engender. Because even the most sophisticated visual simulation systems cannot render scenes with complete fidelity, it is critical to identify rendering simplifications that can be made with minimal impact on perceptual realism.

In a series of three experiments, we examined observers' relative sensitivity to anomalies in the rotational and translational components of motion parallax, varying both object structure and depicted distance from the observer to the target. We used objects with complete radial symmetry (i.e., cones) and asymmetric objects that undergo boundary deformation during rotation (i.e., oblong boxes). The stimuli were designed to remove additional, non-motion-rate cues to translational anomalies (e.g., ground plane texture accretion-deletion and anomalous dynamic occlusions). This allowed for a fair comparison of observers' sensitivities to levels of distortion introduced in the rotational and translational components of motion parallax.

\section{Experiment 1: Rotational Anomalies}

In the first experiment, we examined observers' sensitivity to the rotational component of motion parallax. This was done by assessing their ability to identify a target object with an anomalous rotation (i.e., too fast or too slow for its depicted depth) in a field of distractor objects with canonical rotations. We chose this "odd-man out" paradigm to reflect the fact that database designers frequently mix billboarded and properly rendered 3-D objects; thus, we wanted to establish how discernible an object's noncanonical rotation is in an otherwise canonical scene.

\section{Method}

Participants. Twelve men were paid participants in this experiment. They ranged in age from 18 to 25 years; all had $20 / 20$ or corrected-tonormal vision. None was informed of experimental hypotheses.

Apparatus and stimuli. The stimuli were generated by a high-power graphics computer (Silicon Graphics Onyx RE2; Mountain View, CA) and displayed on a 19 -in. $(48.25-\mathrm{cm})$ diagonal, highresolution color monitor with a pixel resolution of $1280 \times 1024$. Participants viewed the monitor from a distance of $68 \mathrm{~cm}$ through a reduction hood attached to the front of the monitor. The reduction hood helped maintain a fixed viewing distance and, through a series of baffles, removed screen surface reflections. The viewable portion of the monitor through the viewing hood subtended a horizontal angle of $19^{\circ}$. Observers responded by pressing keys on a standard alpha-numeric keyboard that lay on a table near their right hands.

Each trial consisted of a 20 -s animation depicting lateral eye-point translation over a ground plane with 12 objects on it. In the course of the animation, the observer's eye point translated 330 graphical units (gu) to the left at a constant rate of $16.5 \mathrm{gu} / \mathrm{s}$. The animation was updated at 30 frames per second, synchronized to the monitor's $60-\mathrm{Hz}$ 
refresh cycle. The depicted viewing volume was minified so as to provide a $60^{\circ}$ horizontal field-ofview in the $19^{\circ}$-viewing aperture (this is analogous to viewing a scene through a wide-angle lens). Given this graphical scaling, an object 18-gu wide at a depth of $100 \mathrm{gu}$ would subtend $4.77^{\circ}$ in the virtual world and be minified to $1.60^{\circ}$ on the image screen. A plan-view schematic of the stimulus layout is shown in Figure 2.

Cones were used as stimulus objects for half of the observers; oblong boxes were used for the other half. The cones were $20 \mathrm{gu}$ high with base diameters of $18 \mathrm{gu}$. The boxes were $9 \mathrm{gu}$ high, $9 \mathrm{gu}$ along one side, and $18 \mathrm{gu}$ along the other. The cones were light green, and each was textured with the same random pattern of 50 white dots. The boxes had three colored textures with a total of 52 white dots on the top and sides. The two short ends were blue, the top was light pink, and the two longer vertical sides were green. The boxes were positioned with their short faces perpendicular to the ground plane; the azimuth orientation of each box's major axis was varied randomly. The ground plane had brown Gouraud-shaded trimesh polygons arranged to produce a pseudo-sine wave luminance pattern along the $x$ axis (i.e., parallel to the eye-point motion), and discrete, evenly spaced strips along the $z$ axis (i.e., orthogonal to the eye-point motion). When a polygon (in this case, a triangle) is Gouraud shaded, different color values are specified for each vertex and then color is smoothly transitioned between vertices across the face of the polygon. This smooth shading provided a compelling perspective view and an unambigu- ous placement of objects in depth without creating discrete surface elements. The remainder of the scene was sky blue. The trial number in each block was displayed in red at the top of the screen. Each cone or box had a dark blue identification number floating just above its apex or corner.

The scene was drawn with normal linear perspective with Graphics Library software (Silicon Graphics). This graphics tool defines a linear perspective view with a specified field of view $\left(60^{\circ}\right)$, an aspect ratio (i.e., window width $\times$ window height, which we set to 1.0 ), and near and far clipping planes. Although the metric unit (graphical units) used in these graphic settings may seem arbitrary, the resultant geometry is canonical; the apparent sizes of the objects on screen at different graphical distances are related by the tangent function, as in the real world. Thus, the stimuli possessed many static depth cues (e.g., relative size, height in picture plane, and texture density) to inform observers of depicted depth. The observer's eye point was positioned $50 \mathrm{gu}$ from the near edge of the plane and elevated slightly (10 to $25 \mathrm{gu}$ ) above the plane. One frame of a cone stimulus animation is shown in Figure 3.

In each trial, one target cone (or box) was rotated anomalously at one of six distortion levels. These levels in percentage reduction of canonical rotation were $-150 \%,-100 \%,-50 \%, 50 \%, 100 \%$, and $150 \%$. The negative numbers indicate that the distortion was an increase in canonical rotation. Thus, a target with $-100 \%$ distortion would rotate at twice its canonical rate. A target with $100 \%$ distortion would not rotate during the eye point

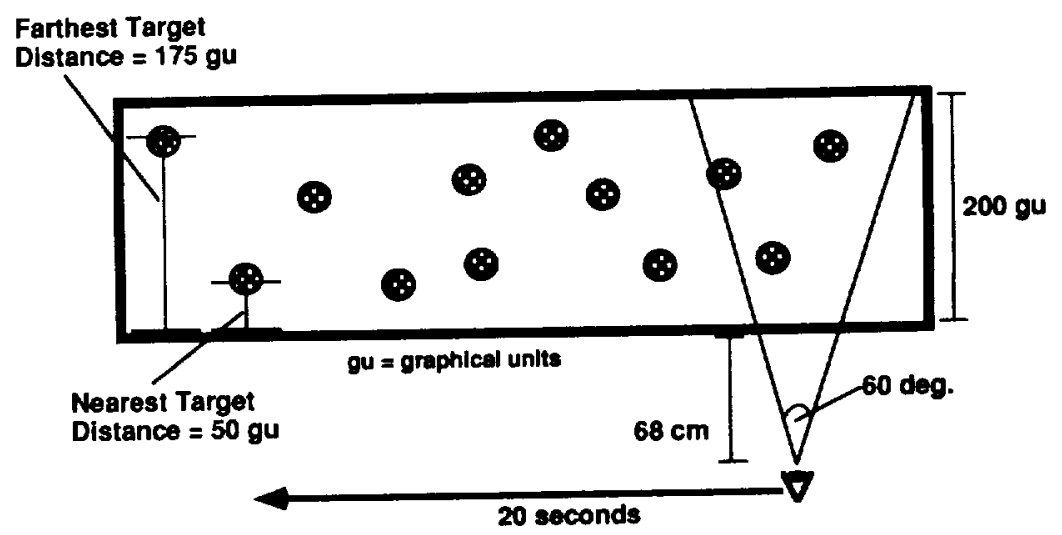

Figure 2. A plan-view schematic of the stimulus layout in the three experiments. The 12 objects were either cones (shown) or oblong boxes. Placement of the objects varied from trial to trial, as did the selection of the target. deg. = degrees. 


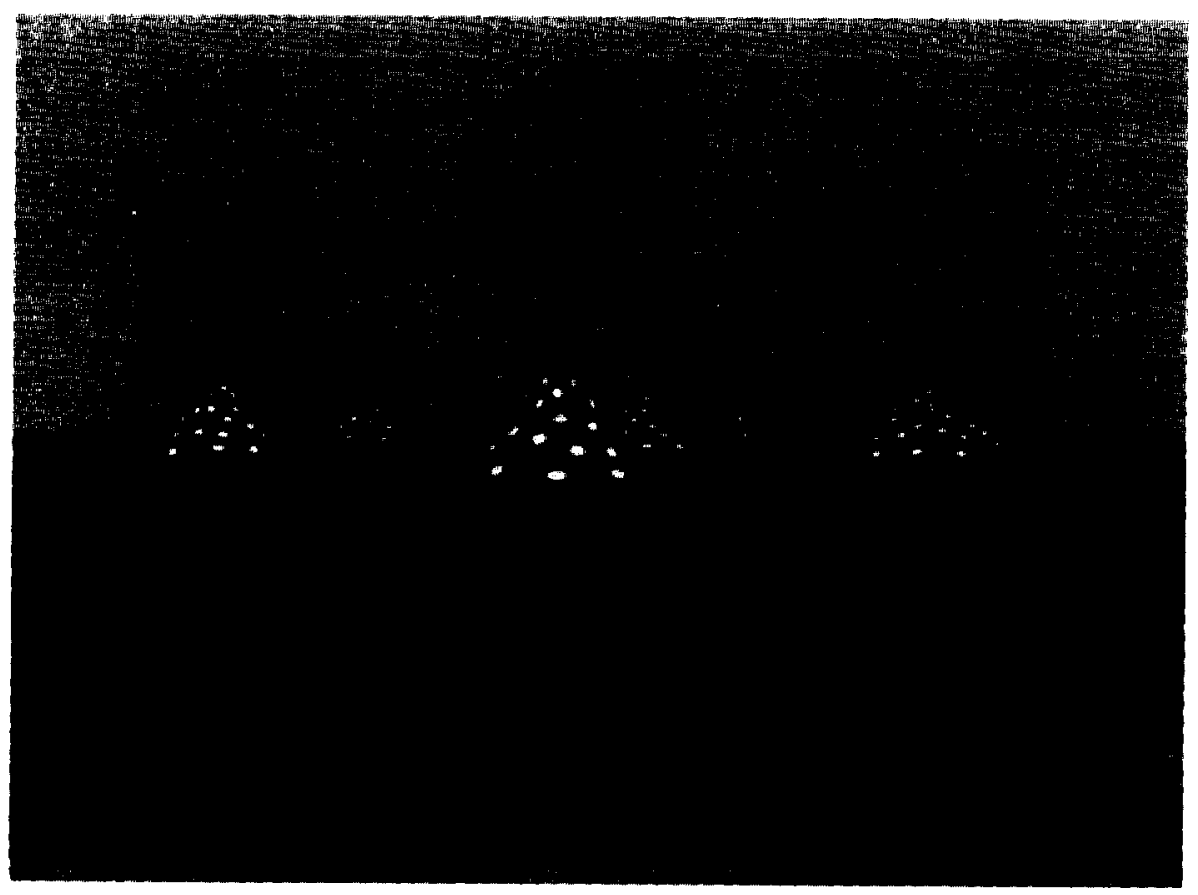

Figure 3. Sample frame from one of the cone stimulus animations. The images in the experiments were in color.

displacement; this is the situation when a graphical object is billboarded. In addition, targets could be placed at six distances from the perspective view eye point as defined by the graphics environment: $50,75,100,125,150$, and 175 gu along the $z$ axis from the near edge of the ground plane.

Design. The experiment had a mixed design with level of distortion, sign of the distortion, and distance from the observer as within-observer variables, and the two types of stimulus objects as a between-groups variable. The performance measure was the number of trials in which the anomalously rotating target was correctly identified.

Procedure. Observers were told that they would be involved in an experiment of simulated visual motion. The scene was compared with what one might see looking out the window of a moving train. The experimenter described the rotational and translational effects that occur during motion parallax. Observers were then told that they would be looking for a single cone or box that was rotating anomalously (i.e., "too fast or too slow for the depth it is at").

The participants were then given 18 practice trials. The first series of four trials demonstrated extreme distortions $( \pm 200 \%)$, and the experi- menter pointed out the target. This was to ensure that participants had a clear understanding of the anomaly they were supposed to identify. Observers were then shown a set of 12 practice trials comprised of two examples of each level of rotational distortion to demonstrate the range of distortions in actual experimental trials. It was explained that this was the only block of trials where there would be a mix of distortion magnitudes and that the following six blocks of experimental trials would each have a single level of distortion. Observers were told to press the keyboard key labeled with the number ( 1 through 12) that corresponded to the object they thought was rotating anomalously. Responses were permitted only after the animation was complete (as signaled by a beep from the computer). Observers were instructed to guess if they wcre unsure of the target, as the experiment would not continue without a response after each trial. Observers were encouraged to respond at their own pace and to pause, if need be, between trials by delaying their response. Furthermore, they were instructed to look away from the display if at any time they experienced vertigo or nausea.

A typical trial went thus: An audible tone sounded, and the animation began. When the 
animation terminated, there was another audible tone indicating that the observer should respond; the observer responded and the screen immediately shifted to a gray mask for $1.5 \mathrm{~s}$. After this, there was an audible tone to indicate the start of a new trial. Six blocks of 30 trials were administered with the order of distortion level counterbalanced across observers in a pseudo-Latin-squares design (i.e., the order was randomly generated for the first observer and rotated one step for each subsequent observer).

\section{Results}

A $6 \times 3 \times 2$ within-observer analysis of variance (ANOVA) was performed independently on the cone and box data to assess the effects of target distance, distortion magnitude, and distortion sign. For the observers shown the cones stimuli, performance was significantly affected by distortion magnitude, $F(2,10)=62.95, p<.001$, with performance improving with distortion magnitude (see Figure 4). In addition, there was a significant effect of simulated distance from eye point, $F(5,25)=$ $13.68, p<.001$, with performance degrading with increasing distance (see Figure 5). Trend analyses indicated that the linear component of these effects accounted for most of the variance $(94 \%$ of the distortion magnitude effect and $93 \%$ of the distance effect). There was a small but significant effect for the sign of the distortion, $F(1,5)=6.67$, $p=.049$; performance was slightly better for

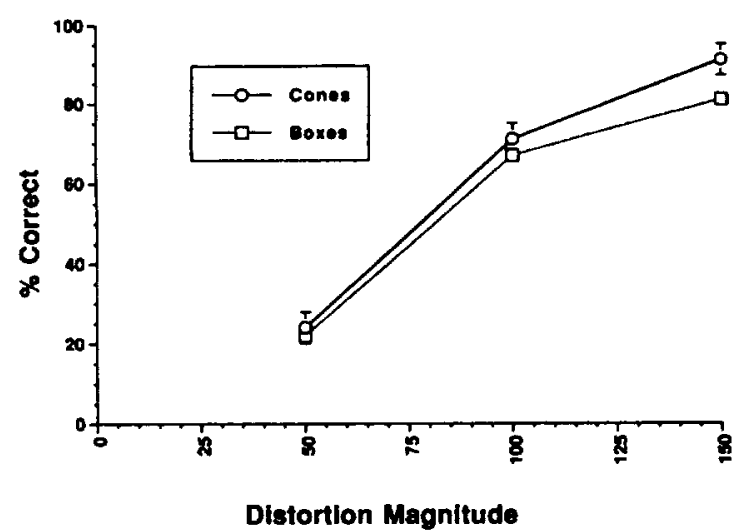

Figure 4. Percentage of correct responses averaged across observers in Experiment 1 as a function of object type (cones or boxes) and distortion magnitude (collapsed over sign). The error bars show $\pm 1 S E$ for the cone data. The box data had similar spreads.

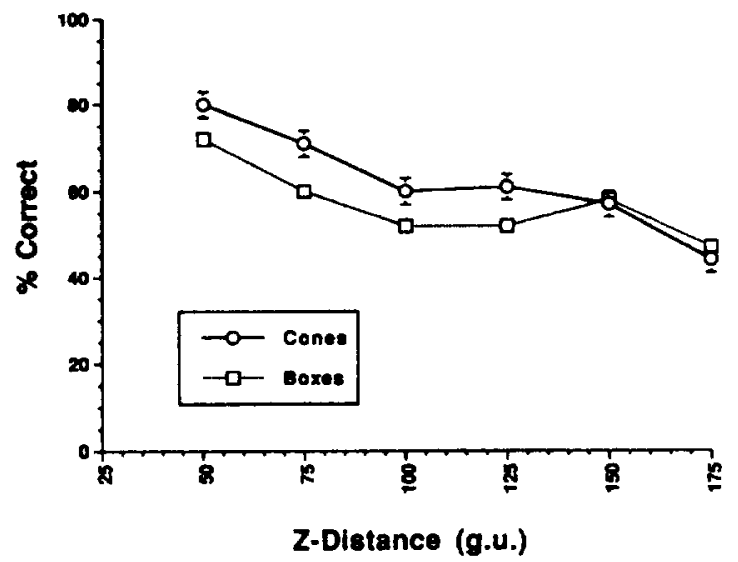

Figure 5. Percentage of correct responses averaged across observers in Experiment 1 as a function of object type and target distance. Error bars show $\pm 1 S E$ for the cone data. The box data had similar spreads. g.u. = graphical units.

negative distortions (i.e., increased rotational rates). There were no significant two- or three-way interactions.

When queried about their assessment strategy, observers commented that they consciously assessed the rotational component of each cone individually. None of the observers described the distortion as obvious on casual inspection or when looking globally at the field of candidates.

For the box stimulus group, the variables affecting performance were distortion magnitude, $F(2$, $10)=68.89, p<.001$, and simulated distance from the eye point, $F(5,25)=7.27, p<.001$. The functional relationship of these variables was the same as with the cones: performance improved with increasing distortion and was worse with increasing distance (Figures 4 and 5). Trend analyses revealed that the functions describing the distortion magnitude and distance variables were primarily linear in nature, accounting for $92 \%$ and $66 \%$ of the variance, respectively. As with the cone data, there were no significant interactions.

Observers' descriptions of their strategies were similar to the cone stimulus group. For the most part, individual assessment of each box was necessary to determine whether it was rotating anomalously.

A between-group ANOVA comparing the performance of the box stimulus group to the cone stimulus group revealed no significant difference. For both conditions, the $50 \%$ detection threshold 
for rotational anomaly was estimated at a magnitude of roughly $80 \%$ distortion, as shown in Figure 6. Although the probability of choosing a correct target from a set of 12 by chance is 0.085 , we used the 0.50 performance level for two reasons: First, objects near the edges were not allowed to be targets (because they were visible for only a brief time interval), so the actual set of candidate targets was smaller than 12 . Second, $50 \%$ detection seemed a reasonable criterion level for classifying the rotational anomaly as a potentially annoying artifact in a simulation display.

\section{Experiment 2: Translational Anomalies}

The second experiment examined observers' sensitivity to distortions in the translational component of motion parallax. This was done with the same paradigm as Experiment 1: observers identified a target object with an anomalous translational rate from a field of distractors with canonical translational components.

\section{Method}

Participants. Participants were 12 men ranging in age from 18 to 25 . All had vision that was $20 / 20$ or corrected to normal; none participated in the first experiment. Observers were paid.

Apparatus and stimuli. The stimuli were the same as in Experiment 1, except that the target object demonstrated a translational rather than rotational anomaly. Thus, one cone or box trans- lated either too fast or too slow for its depth. As described earlier, the shading gradient along the axis of translation consisted of gradual modulation, such that no accretion-deletion of ground surface texture or edges was apparent during the anomalous translation. The levels of distortion were smaller than in Experiment 1 because pilot studies demonstrated translational anomalies to be far more salient than rotational ones. Distortion levels were $-50 \%,-35 \%,-20 \%, 20 \%, 35 \%$, and $50 \%$. Negative numbers indicate an increase in the speed of translation. Targets were placed at the same $z$-axis distances as in Experiment 1, but placement was constrained to prevent what we term "anomalous occlusions." Such occlusions occur if the target overtakes a closer object (or is overtaken by a more distant object). Such clear violations of normal dynamic occlusion could potentially provide a strong cue to identify the target. To avoid such extraneous information, we defined zones about the target in which no objects could be placed. An example of such a "no-cone zone" is shown in Figure 7.

Design. The design was the same as in Experiment 1 , with level of distortion, sign of distortion, and simulated distance as within-observer variables, and object type (cones or boxes) as a between-groups variable.

Procedure. The procedure was the same as in Experiment 1. The first set of demonstration trials (i.e., those demonstrating extreme anomalies) had distortion magnitudes of $\pm 75 \%$. As in Experiment 1 , six blocks of 30 trials were presented, with the

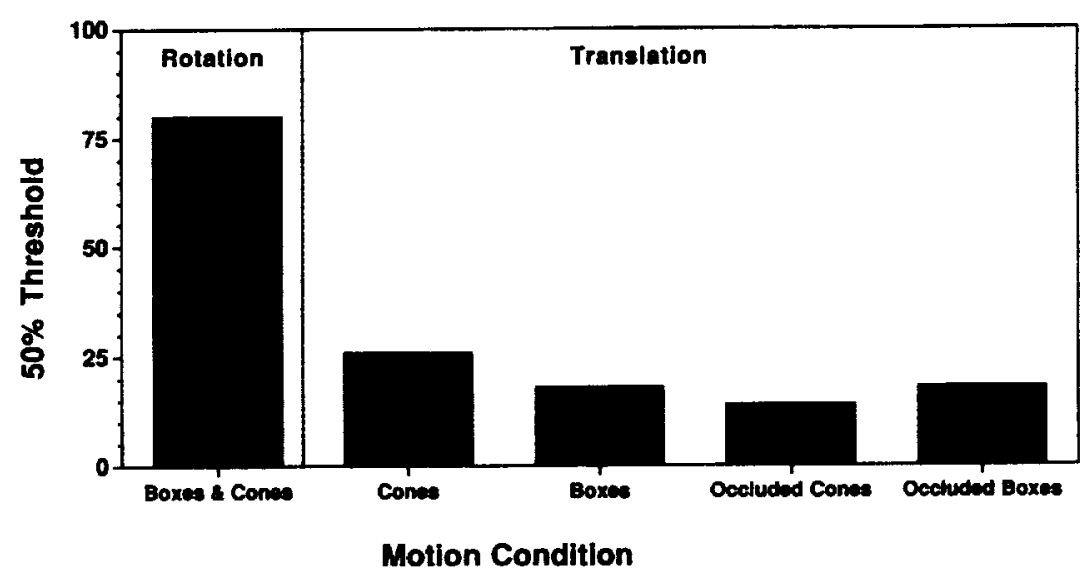

Figure 6. Thresholds for $50 \%$ target detection for the three experiments. Because there was no difference in threshold estimates, a single value is shown for both cones and boxes in Experiment 1. 


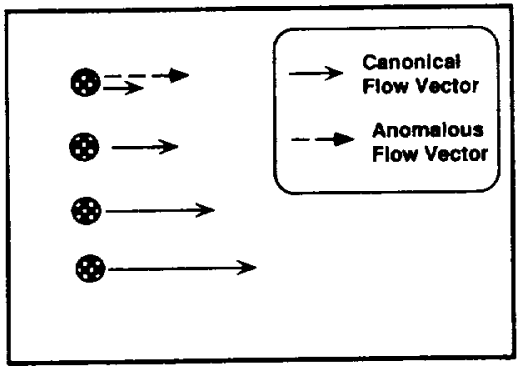

Anomalous Occlusion Occurs

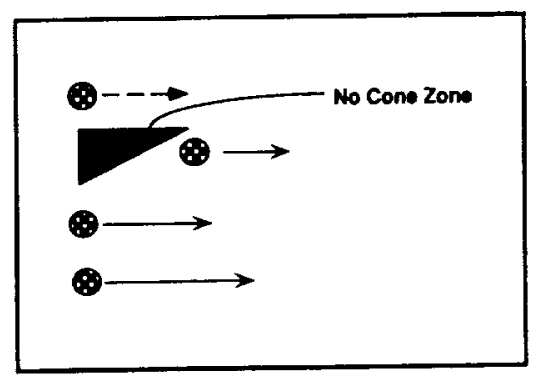

No Anomalous Occlusion Occurs

Figure 7. No-cone zone for an anomalous translation stimulus in Experiment 2. Because of the increase in the translational vector from the experimental distortion, the target cone overtook an object more proximal to the observer. To avoid the extraneous cue provided by such anomalous dynamic occlusions, we constrained object placement such that none fell where such events would occur.

order of distortion magnitude counterbalanced across observers.

\section{Results}

The data were analyzed with the same statistical methods as in Experiment 1. For the group viewing the cones stimuli, there was no effect for sign of distortion. There were significant effects for both distortion magnitude and target distance. Performance again improved as a function of increasing magnitude of distortion, $F(2,10)=65.22, p<$ .001 , and varied as a function of simulated distance from the observer, $F(5,25)=5.41, p<.01$. These effects are shown in Figures 8 and 9. Trend analyses revealed that the performance function

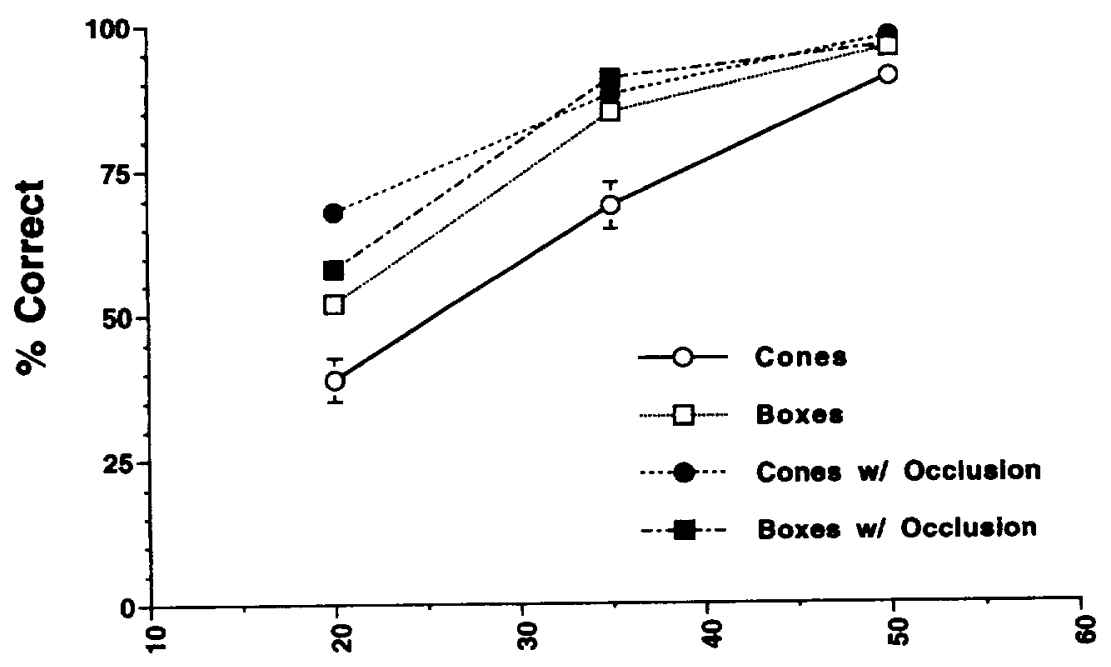

\section{Distortion Magnitude}

Figure 8. Percentage of correct responses averaged across observers in Experiments 2 and 3 as a function of target type and distortion magnitude (collapsed over sign). Open symbols show results from Experiment 2, where anomalous occlusions were prevented; filled symbols show results from Experiment 3, where anomalous dynamic occlusions were allowed. Error bars show $\pm 1 S E$ for the cone-without-occlusion data. Other data sets had similar spreads. 


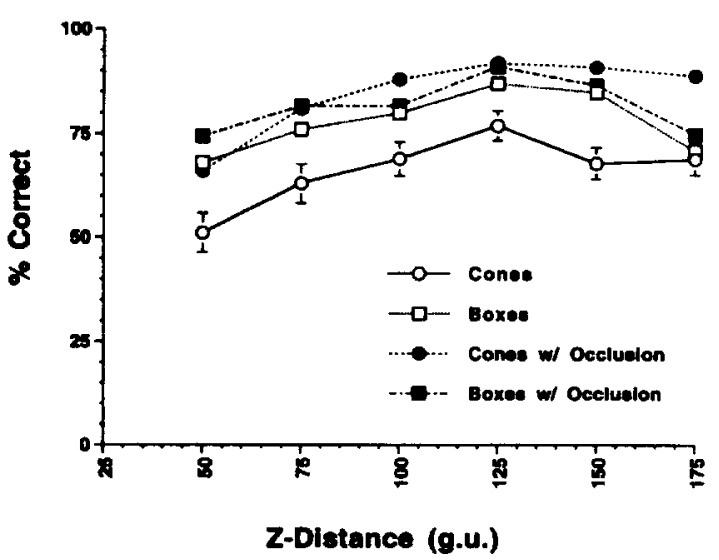

Figure 9. Percentage of correct responses averaged across observers in Experiments 2 and 3 as a function of target type and target distance. The coding convention is the same as in Figure 7. Error bars show $\pm 1 S E$ for the cone-without-occlusion data. Other data sets had similar spreads. g.u. = graphical units.

related to the magnitude of distortion could be described as a primarily linear effect (accounting for $99 \%$ of the total variance). The strongest component of the distance effect was quadratic, accounting for $41 \%$ of the variance. No significant interactions were revealed.

Results for the box stimulus condition were similar to the cone stimulus condition. Again, there was significant improvement in performance associated with increases in distortion magnitude, $F(2,10)=49.75, p<.001$ (the linear component accounting for $93 \%$ of the variance). There was also a significant effect for target distance, $F(5,25)=6.13, p<.01$ (the quadratic component accounted for $49 \%$ of the variance). There were no significant interactions. These results are illustrated in Figures 7 and 8.

Comparison of the two groups indicates that performance was significantly better in the box stimulus condition, $F(1,10)=8.17, p<.05$. The $50 \%$ threshold for observers in the box condition was roughly $18 \%$, whereas threshold for those in the cone condition was approximately $26 \%$ (see Figure 6).

In both groups, observers described their strategy as global. That is, they reported that they attended to the entire field, and the anomalous object "popped out" at them.
Experiment 3: Translational Anomalies With Anomalous Occlusions

The stimuli in the second experiment were explicitly designed to avoid anomalous dynamic occlusions involving the target and distractor objects. In principle, such violations of nominal occlusion relations should be a strong cue for target identification. In this experiment, we allowed anomalous occlusions to occur and assessed the impact of this cue on target identification. Anomalous occlusions occurred whenever targets translating too slowly had objects behind it (i.e., in all but the 175-gu distance case) or targets translating too quickly had objects in front of it (i.e., in all but the 50 -gu distance case).

\section{Method}

Participants. Twelve men, ranging in age from 18 to 25 , were paid to participate in this experiment. None had taken part in the previous experiments or were aware of the experimental hypotheses.

Apparatus and stimuli. The stimuli were the same as in Experiment 2, except that anomalous occlusions were permitted. As previously described, anomalous occlusions occur when a more distant object overtakes a closer object in the direction of eye point movement. Clearly, such occlusions do not occur during normal motion parallax.

Design. The design was the same as that in Experiments 1 and 2 . Level of distortion, sign of distortion, and simulated distance from the observer were within-observer variables, and type of objects (cones or boxes) was a between-groups variable. The distortion magnitude levels were the same as those used in Experiment 2.

Procedure. The procedure was the same as that used in Experiments 1 and 2. The number of trials and blocks and the method of counterbalancing distortion level was the same as in the previous experiments.

\section{Results}

The data were analyzed with the same methods as in Experiments 1 and 2. For the cone stimulus group, there was no effect for sign of distortion. 
There were significant effects for distortion magnitude, $F(2,10)=21.28, p<.001$, and target distance, $F(5,25)=8.46, p<.001$. These results are shown in Figures 8 and 9. Trend analyses revealed that the distortion magnitude effect was primarily linear (accounting for $96 \%$ of the variance); the distance effect contained significant linear and quadratic components $(67 \%$ and $32 \%$ of the variance, respectively). No significant interactions were noted.

The results were similar for the box stimulus group, as shown in Figures 8 and 9. The sign of distortion had no significant effect. Distortion magnitude had a significant effect, $F(2,10)=$ $45.30, p<.001$, where linear component accounted for $86 \%$ of the total variance. Target distance produced a smaller, yet significant effect, $F(5,25)=3.98, p<.01$, with a dominant linear component (accounting for $71 \%$ of the variance). Analyses revealed no significant interactions.

There was no significant difference in performance between the observers who viewed cone stimuli and those who viewed box stimuli. Comparing observers' performance in Experiment 2 (no anomalous occlusions) to Experiment 3 (anomalous occlusions), there was no significant difference for the two translating box conditions, but there was a significant difference between the two translating cone conditions, $F(1,10)=15.18, p<$ .01 , with better target identification when anomalous occlusions occurred. This difference can be seen in the threshold levels shown in Figure 6.

\section{Discussion}

Observers were much more sensitive to distortions of the translational component of motion parallax than to rotational anomalies. Detection thresholds were approximately four times greater for rotational distortions. This insensitivity to rotational distortion was virtually equivalent for objects with and without radial symmetry; boundary deformations of nonsymmetric objects did not significantly aid recognition of rotational distortions. Thus, our findings suggest that billboarding may prove an effective technique for a broader range of object classes than has been heretofore supposed.

The efficacy of billboarding is likely to vary as a function of the distance from the object to the observer; for both object types, recognition of distortions decreased as distance increased. (The rotational rate of more distant objects is, of course, smaller; distortions were defined proportionally.) Figure 10 shows the subset of Experiment 1's data that involved billboarded stimuli; these were the targets with $+100 \%$ distortion (i.e., the rotational component removed). As shown, target identification diminishes as distance increases, with performance consistent at about a 50\% target detection rate at distances of $100 \mathrm{gu}$ and beyond. Distortion detection may be even lower if observers are engaged in other tasks with the database (e.g., simulated flight), rather than our experimental task which focused on anomaly identification. Whether this distance is best characterized as a function of eye heights or object size (or both) requires further investigation.

Regardless of the units, such metrics would have utility in defining the distance at which billboarding is more or less likely to be noticed. The visual database manager could then swap between billboarded and 3-D versions of the object as required, much as current systems swap among various level of detail renderings as a function of viewing distance (and other metrics; Funkhouser \& Sequin, 1993). Although further research is needed to determine if special consideration must be given to objects that reveal noticeable emergent features during rotation (e.g., the face of a head), our findings provide psychophysical support for

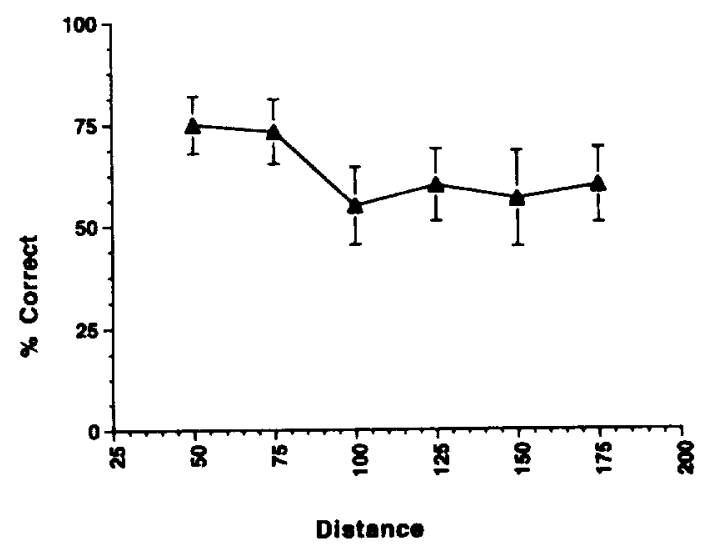

Figure 10. Subset of Experiment 1's data corresponding to a billboarding situation (i.e., the rotational component of motion parallax is removed). Performance is averaged across observers and object type. Error bars show $\pm 1 S E$. 
graphical techniques that achieve computational savings by deleting or simplifying ( $R$. Pausch, April 14,1995 , personal communication) rotational transformations.

Observers' relative insensitivity to the rotational component of motion parallax might be related to the small magnitude of displacements that occur during object rotations. Consider, for example, a target cone at a depth of $100 \mathrm{gu}$ with a $50 \%$ distortion. Given the eye-point translation rate of our displays ( $330 \mathrm{gu}$ in $20 \mathrm{~s}$ or $16.5 \mathrm{gu} / \mathrm{s}$ ), a $50 \%$ translational distortion would add or subtract a motion component of $8.25 \mathrm{gu} / \mathrm{s}$. The analogous rotational distortion would be only $0.67 \mathrm{gu} / \mathrm{s}$ at most (i.e., an element at the base of the cone would have its instantaneous tangential velocity altered by that amount in a $50 \%$ distortion). Thus, although the rotational and translational components are mathematically equivalent in terms of angular displacement, the Euclidean (and hence image-plane) displacement for a given angle is far larger for the translational component. Whereas the psychophysical literature suggests fairly robust sensitivity to angular velocity changes (Kaiser, 1990; Kaiser \& Calderone, 1991), the low rates of rotation that usually occur during motion parallax might stress the visual system's sensitivity.

People's relative insensitivity to detecting object rotations during self-motion has implications for the derivation of an object's 3-D structure from motion parallax. In principle, the extraction of a stationary object's 3-D structure during selfmotion has an advantage over observing the object undergoing a similar amount of effective rotation. The advantage is that when a translating observer views a stationary object, its effective rotation is given in its observer-relative angular displacement; when viewing a similarly small object rotation, the angle of effective rotation is difficult to discern (Proffitt, Rock, Hecht, \& Schubert, 1992). On the other hand, there are numerous reports that depth from motion parallax and depth from object rotation are processed similarly, even when the motion parallax is produced by self-motion (Braunstein, Liter, \& Tittle, 1993; Caudek \& Proffitt, 1993). Others (e.g., Ono \& Steinbach, 1990) report some advantage for viewer-produced motion parallax. One reason for the similarity between the processing of depth from object rotations and motion parallax may relate to the difficulty entailed in determining whether an object is itself rotating when observed by a translating observer. If the object is independently rotating, then observerrelative rotation is not informative, and the event may best be processed as if it were an object rotation.

More broadly, our findings suggest that for motion parallax observers are not equally sensitive to all informational components. This appears to be true despite the fact that in principle the salient and nonsalient components contain information of equal mathematical validity; both the rotational and translational components of motion parallax invariantly specify the depth ratios of objects in the visual field. Our findings demonstrate a selective sensitivity of the perceptual system to these various sources of information and suggest ways in which computer-rendering techniques can exploit these insensitivities to achieve computational savings (Kaiser \& Proffitt, 1992).

\section{References}

Braunstein, M. L., Liter, J. C., \& Tittle, J. S. (1993). Recovering three-dimensional shape from perspective translations and orthographic rotations. Joumal of Experimental Psychology: Human Perception and Performance, 19, 598-614.

Caudek, C., \& Proffitt, D. R. (1993). Depth perception in motion parallax and stereokinesis. Joumal of Experimental Psychology: Human Perception and Performance, 19, 32-47.

Foley, J. D., van Dam, A., Feiner, S. K., \& Hughes, J. F. (1990). Computer graphics: Principles and practice (2nd ed.). New York: Addison-Wesley.

Funkhouser, T. A., \& Sequin, C. H. (1993). Adaptive display algorithms for interactive frame rates during visualization of complex virtual environments. Computer Graphics Proceedings, Annual Conference Series, 247-254.

Kaiser, M. K. (1990). Angular velocity discrimination. Perception \& Psychophysics, 47, 149-156.

Kaiser, M. K., \& Calderone, J. B. (1991). Factors influencing perceived angular velocity. Perception \& Psychophysics, 50, 428-434.

Kaiser, M. K., \& Proffitt, D. R. (1992). Using the stereokinetic effect to convey depth: Computationally efficient depth-from-motion displays. Human Factors, 34, 571-581.

Ono, H., \& Steinbach, M. J. (1990). Monocular stereop- 
sis with and without head movement. Perception \& Psychophysics, 48, 179-187.

Proffitt, D. R., Rock, I., Hecht. H., \& Schubert, J. (1992). The stereokinetic effect and its relation to the kinetic depth effect. Joumal of Experimental Psychology: Human Perception and Performance, 18, 3-21.

von Helmholtz, H. (1962). Treatise on physiological optics
(Vol. 3). (J. P. C. Southall, Ed. \& trans.). New York: Dover. (From the 3rd German ed., 1910.)

Received December 1, 1994 Revision received May 8, 1995 Accepted June 28, 1995 
